\title{
Discontinuation of tyrosine kinase inhibitors in chronic myeloid leukemia: when and for whom?
}

\author{
Ehab Atallah ${ }^{1}$ and Charles A Schiffer ${ }^{2}$ \\ ${ }^{1}$ Medical College of Wisconsin and ${ }^{2}$ Karmanos Cancer Institute, Wayne State University \\ School of Medicine, Milwaukee, WI, USA
}

Haematologica 2020

Volume 105(12):2738-2745

\section{Correspondence: \\ EHAB ATALLAH \\ eatallah@mcw.edu \\ CHARLES A SCHIFFER \\ schiffer@karmanos.org}

Received: July 10, 2020.

Accepted: September 17, 2020.

Pre-published: October 9, 2020.

doi:10.3324/haematol.2019.242891

(C)2020 Ferrata Storti Foundation

Material published in Haematologica is covered by copyright. All rights are reserved to the Ferrata Storti Foundation. Use of published material is allowed under the following terms and conditions:

https://creativecommons.org/licenses/by-nc/4.0/legalcode. Copies of published material are allowed for personal or internal use. Sharing published material for non-commercial purposes is subject to the following conditions:

https://creativecommons. org//licenses/by-nc/4.0/legalcode, sect. 3. Reproducing and sharing published material for commercial purposes is not allowed without permission in writing from the publisher.

\section{ABSTRACT}

reatment discontinuation is considered one of the main goals of therapy for patients with chronic myeloid leukemia. Several criteria are felt to be necessary to consider discontinuation, while others may predict a better chance of achieving treatment-free remission. Criteria for discontinuation include patients in chronic phase chronic myeloid leukemia, a minimum duration of tyrosine kinase inhibitor therapy of 3 years, sustained deep molecular response for at least 2 years and a molecular response of at least MR4. In addition, proper education of the patient on the need for more frequent monitoring, possible side effects related to stopping and having a reliable real-time quantitative polymerase chain reaction laboratory are paramount to the safety and success of treatment-free remission. Realistically though, a maximum of only $20-30 \%$ of newly diagnosed patients will be able to achieve a successful treatment-free remission. In this article we will review for whom and when a trial of discontinuation should be considered.

\section{Introduction}

Although treatment with tyrosine kinase inhibitors (TKI) has led to remarkable improvement in the overall survival of patients with chronic myeloid leukemia (CML), until recently these drugs were not considered curative as patients needed to be treated indefinitely. Hematopoietic stem cell transplantation was considered to be the only curative therapy for patients with CML. Over the last decade, several studies have demonstrated that a proportion of patients may be able to stop TKI therapy without relapse, potentially rendering them cured of their disease. ${ }^{1.7}$ After discontinuing TKI therapy, the levels of $B C R-A B L 1$ in the peripheral blood should be monitored closely by polymerase chain reaction (PCR), especially in the first year, and monitoring should continue indefinitely. This approach has been safe, with essentially all patients with a molecular relapse responding to re-initiation of TKI therapy and only a few case reports of disease progression to accelerated phase or blast crisis among thousands of patients discontinuing therapy. This rare phenomenon of sudden transformation to accelerated phase or blast crisis has also been reported in responding patients who remain on TKI. ${ }^{8}$ With discontinuation, approximately $50 \%$ of patients will stay in remission and will not need to restart therapy.

This goal of being off drug therapy, also known as treatment-free remission (TFR), is emerging as the new aim of CML therapy. TFR has several benefits for patients, including improved quality of life, reduced cost to patients and society and decreased risk of long-term complications. Our aim in this article is to review the results of trials on stopping TKI in patients with CML and to discuss some clinical and biological questions raised by the observations.

\section{Who is eligible to stop therapy?}

Current guidelines, ${ }^{9,10}$ recommend considering stopping therapy for patients in chronic phase CML, who have been on TKI therapy for at least 3 years, and who have had a continued deep molecular response (DMR) lasting at least 2 years, with DMR defined as a $B C R / A B L 1$ level of $<0.01 \%$ on the international scale (IS) (equivalent to a 4-log reduction in transcript level from baseline, MR4). Studies have only 
included patients with chronic phase CML. The possibility of stopping therapy in patients with prior accelerated phase or blast phase is unknown at present. In general, we do not recommend stopping drug in such patients given the lack of data.

The depth and duration of response that need to be achieved prior to discontinuation remain unclear. The required duration of DMR has varied from 1 to 3 years in different studies (Table 1). Most studies enrolled patients who were in a continued DMR for at least 2 years and the duration of DMR has been predictive of successful TFR (see later). Initially, studies enrolled only patients with undetectable $B C R-A B L 1$ by PCR with an assay sensitivity of at least a $5 \log$ reduction. ${ }^{11}$ This was followed by other trials such as the EURO-SKI ${ }^{6}$ and LAST studies ${ }^{12}$ in which patients with $B C R / A B L 1<0.01 \%$ were enrolled. In the Euro-SKI trial, there was no difference in relapse rate between patients who had IS $\%<0.01 \%$ or $0.0032 \%{ }^{13}$ In contrast, the DESTINY trial conducted in the United Kingdom, enrolled patients with BCR-ABL1 $<0.1 \%$ (defined as a major molecular response, MMR) and the rate of successful TFR only for patients in MMR was lower $(33 \%)$. Based on that, a level of $0.01 \%$ or MR4 is considered an appropriate level to consider discontinuation outside of clinical trials.

Of note, we and others have described that a significant number of patients who meet stopping criteria are actually not interested in stopping TKI therapy. These observations were made at a time when stopping TKI was still considered experimental and guidelines did not mention this option. Patients were hesitant to stop taking a drug mainly on the principle of "if it ain't broke, don't fix it". $.4,15$ In particular, patients who were not experiencing side effects from the TKI and who had good insurance coverage, so that out-of-pocket costs were minimal, had less obvious motivation to consider discontinuation. In contrast, more recently, many patients who have been told of their "excellent" responses have read about TFR on the Internet and are inquiring about stopping therapy, some- times after relatively short exposure to treatment. Hence, the need for physicians to understand the implications of different levels of response on the IS as well as the requirement for many years of treatment for most patients.

Although most trials only enrolled patients with no history of resistance to TKI, some patients who were resistant to frontline imatinib may stop TKI treatment successfully.,16-18 The DASfree study enrolled patients on first- or second-line treatment with dasatinib.? Of the 84 patients enrolled, 37 (44\%) and 47 (56\%) were receiving dasatinib as first- and second-line treatment, respectively. At 24 months, the rate of TFR was $51 \%$ and $42 \%$ in those treated with dasatinib first-line or as a subsequent line. The rate of successful TFR was $44 \%$ for patients who were either resistant or intolerant to first-line dasatinib. The ENEStop study enrolled patients intolerant of or resistant to imatinib. Of the 163 patients enrolled, 38 were resistant to imatinib. After 1 year of nilotinib consolidation therapy, 30 patients moved on to the TFR phase, of whom 16 $(53 \%)$ remained off drug at 48 weeks ${ }^{16,19}$ (Table 2).

A second attempt at TFR may be considered in some patients. ${ }^{20-22}$ The RE-STIM trial enrolled patients who had previously relapsed after TKI discontinuation and regained a DMR. ${ }^{21}$ Of the 70 patients, $35 \%$ remained off drug at 36 months. The median duration of TKI therapy after restarting was 5 months (range, 2-42 months). $B C R$ $A B L 1$ kinetics at the time of first discontinuation was most predictive of success of a second TFR. The chances of a successful second TFR at 36 months were $46 \%, 31 \%$ and $0 \%$ for patients who had undetectable transcripts or >MR4.5 versus MR3-MR4.5 versus loss of MMR at 3 months after first discontinuation. In the Treatment-free Remission Accomplished by Dasatinib (TRAD) trial, ${ }^{20}$ patients who had confirmed loss of MR4 or loss of MMR after discontinuing imatinib were started on dasatinib 100 mg daily. Patients who achieved MR4 and sustained this level of response for at least 12 months had a second attempt at TFR. The TFR success rate at 6 months was $21.5 \%$. Factors associated with a successful second TFR

Table 1. Key characteristics of selected studies of discontinuation of tyrosine kinase inhibitor therapy.

\begin{tabular}{|c|c|c|c|c|c|c|c|c|}
\hline \multirow[b]{2}{*}{ Reference } & \multirow[b]{2}{*}{$\begin{array}{c}\text { N. of } \\
\text { patients }\end{array}$} & \multirow[b]{2}{*}{ TKI(s) } & \multicolumn{3}{|c|}{ Elighibility criteria } & \multirow{2}{*}{$\begin{array}{l}\text { Median time } \\
\text { in MR, months } \\
\text { (range) }\end{array}$} & \multirow{2}{*}{$\begin{array}{l}\text { Median } \\
\text { duration on } \\
\text { TKl, months } \\
\text { (range) }\end{array}$} & \multirow[t]{2}{*}{ Trigger to restart TKI } \\
\hline & & & $\begin{array}{c}\text { Duration of } \\
\text { TKI (y) }\end{array}$ & $\begin{array}{c}\text { Depth of } \\
\text { MR }\end{array}$ & $\begin{array}{l}\text { Duration } \\
\text { of MR }(\mathrm{y})\end{array}$ & & & \\
\hline ENESTfreedom ${ }^{4}$ & 222 & Nilotinib & 2 & MR4.5 & 1 & NR & $43.5(32.9-88.7)$ & Loss of MMR \\
\hline STIMl $^{3}$ & 100 & Imatinib\# & 3 & MR5.0 & 2 & 36.4 (24-107) & $58.8(35-112)$ & $\begin{array}{l}\text { At least two positive RT-PCR results } \\
\text { showing an increase of one log or } \\
\text { confirmed loss of MMR }\end{array}$ \\
\hline EURO-SKI ${ }^{6}$ & 868 & $\begin{array}{l}\text { Imatinib or } \\
\text { nilotinib or } \\
\text { dasatinib }\end{array}$ & 3 & MR4.0 & 1 & $56.4(34.8-82.8)$ & 90 (60-118.8) & Loss of MMR \\
\hline DasFree $^{7}$ & 79 & Dasatinib & 2 & MR4.5 & 1 & $28(13-116)$ & $9(29-244)$ & Loss of MMR \\
\hline
\end{tabular}

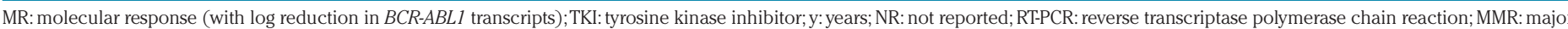
molecular response. *Patient on imatinib only, range not reported.\#Prior interferon therapy allowed. 
were: longer time to molecular relapse after imatinib discontinuation ( $>3$ months $v s .<3$ months), deeper response to initial therapy (5.5 log reduction or deeper vs. others) and loss of MR4 (compared to loss of MMR). ${ }^{20}$ Both studies suggest that patients who have a rapid loss of MMR after a first discontinuation of a TKI have a negligible chance of achieving a seond TFR with TKI therapy alone (Table 3).

It has been suggested that initial treatment with a second-generation TKI, rather than imatinib, might increase the fraction of patients eligible for consideration of stopping therapy because second-generation TKI do lead to a higher percentage of patients achieving MR4 or MR4.5. Whether this actually leads to a higher rate of successful TFR remains unknown as TFR outcomes are not available from the up-front randomized trials evaluating secondgeneration TKI. In the ENESTnd study of imatinib versus nilotinib, the rates of MR4 at 5 years were $42 \%$ and $66 \%$ for patients on imatinib and nilotinib, respectively, while the rates of MR4.5 at 5 years were $31 \%$ and $54 \%$, respectively. ${ }^{23}$ Similarly, in the DASISION study of imatinib versus dasatinib, the rates of MR4.5 were $33 \%$ and $42 \%$, respectively. ${ }^{24}$ However, only about $70 \%$ of patients remain on nilotinib or dasatinib in the long-term and the rates of significant side effects (predominantly cardiovascular and pleural effusions, respectively) are much higher with these drugs than with imatinib. The side effects associated with second-generation TKI may now be lower given better selection of patients, earlier identification of the side effects and the use of lower doses, as will be discussed later. Discontinuation trials in patients treated with nilotinib or dasatinb have shown the same $\sim 50 \%$ rates of TFR as seen in the imatinib trials. ${ }^{7,25,26}$

Another strategy which has been evaluated is to switch imatinib-treated patients to a second-generation TKI to get patients to a deeper response and be eligible for discontinuation. In the ENESTcmr study, patients receiving imatinib who had not achieved MR4.5 were randomized to either imatinib continuation or a switch to nilotinib. ${ }^{27}$ At 24 months, patients on imatinib who had detectable $B C R-A B L 1$ were allowed to switch to nilotinib. At 24 months, the rate of MR4.5 was $54 \%$ for those who switched to nilotinib versus $32 \%$ for those who stayed on imatinib, reiterating prior observations that the depth of response often improves over time in responding patients receiving imatinib. The rate of successful TFR was not reported and a significant fraction of patients $(18 \%)$ discontinued nilotinib because of adverse events, mostly cardiovascular in nature. Of the patients who switched to nilotinib after the 24 months, 37\% achieved MR4.5. It should be noted however, that MR4 is considered sufficient for an attempt at TKI discontinuation, and it is not known whether there were important differences in the rates of sustained MR4 using the two strategies.

In summary, the rates of achieving MR4.5 are approximately $10-20 \%$ higher with second-generation TKI than with imatinib whether the second-generation TKI is started as first-line therapy or switched to later. In addition, second-generation TKI lead to faster and deeper responses which could potentially lead to shorter time on treatment compared to the time on imatinib before a trial of discontinuation. However, this comes at an increased risk of clinical $^{27,28}$ and financial toxicity. ${ }^{29}$ Hypothetically (because prospective data are not available), if 100 patients start imatinib, 40 will achieve MR4. Of those, approximately 20 will have a successful TFR. If a patient starts a secondgeneration TKI, approximately 60 will achieve MR4 and 30 will have a successful TFR.

\section{When to restart?}

In the initial STIM1 ${ }^{11}$ and TWISTER $^{5}$ discontinuation studies, criteria for restarting were loss of MR4.5; i.e., the patient restarted treatment as soon as BCR-ABL1 transcripts were detected. Subsequently, the A-STIM trial

Table 2. Treatment-free remission studies in patients resistant to first-line tyrosine kinase inhibitor therapy.

\begin{tabular}{lcccl} 
& N. of patients & TFR (@months) & TKI & Indication to restart \\
DASfree $^{7}$ & 25 & $40 \%(12)$ & Dasatinib & Loss of MMR \\
ENEStop $^{16}$ & 30 & $26 \%(12)$ & Nilotinib & Loss of MMR or confirmed loss of MR4 \\
\hline DADI $^{17}$ & 13 & $8 \%(36)$ & Dasatinib & Stringent molecular relapse. Restarting at $>0.0069 \%$ IS. \\
2G-TKI $^{18}$ & 9 & $33 \%(12)$ & Dasatinib or nilotinib & Loss of MMR \\
\hline
\end{tabular}

TFR: treatment-free remission; TKI: tyrosine kinase inhibitor; MMR: major molecular response; MR4: molecular response with a 4-log reduction in BCR-ABL1 transcripts; IS: international scale. 2G-TKI: second-generation tyrosine kinase inhibitor.

Table 3. Completed and ongoing studies evaluating a second attempt to achieve treatment-free remission.

\begin{tabular}{lccl} 
& Number of patients & TFR (@months) & Notes \\
TRAD $^{20}$ & 25 & $21 \%(6)$ & Patients restarted dasatinib after MMR loss with imatinib \\
RE-STIM $^{21}$ & 70 & $35 \%(36)$ & $\begin{array}{l}\text { TFR 72\% vs. 36\% for those who did vs. did not remain in DMR at 3 months } \\
\text { with first attempt. 61 of 70 patients received the same TKI }\end{array}$ \\
\hline Matsuki $^{22}$ & 10 & $24 \%(24)$ & All patients on dasatinib. \\
Sweet & 41 & NR & Ongoing study, adding ruxolitinib (NCT03610971) \\
\hline Rousselot & 26 & NR & Ongoing study, adding pioglitazone (NCT02889003) \\
Olsson-Strömberg & 134 & NR & Ongoing study, second attempt with dasatinib (NCT03573596) \\
\hline Spanish & 80 & NR & Ongoing study, second attempt with ponatinib (NCT04160546) \\
ELN & 200 & NR & Ongoing study, second attempt with nilotinib (NCT02917720)
\end{tabular}

TFR: treatment-free remission; MMR: major molecular response; DMR: deep molecular remission; TKI: tyrosine kinase inhibitor; NR: not reported; ELN: European LeukemiaNet. 
demonstrated the safety of restarting treatment at loss of $\mathrm{MMR}^{30}$ and this is the recommended level for restarting in most current guidelines. A number of patients have fluctuating values ranging between 3-4.5 log reductions or undetectable transcripts followed by very low, but detectable values, who do not overtly relapse during long-term follow-up.

It is intriguing that some patients can have detectable $B C R-A B L 1$ transcripts by PCR analysis with no evidence of disease progression. In a study by Ross et al., seven of eight patients with undetectable $B C R-A B L 1$ by real-time quantitative PCR had detectable $B C R-A B L 1$ DNA and none of those relapsed. ${ }^{31}$ One explanation is that the $B C R$ $A B L 1$-positivity arose from non-myeloid lineages such as memory lymphocytes. In a study by Pagani et al., blood from 20 patients in TFR was sorted into granulocytes, monocytes, B cells, T cells and natural killer (NK) cells. $B C R-A B L 1$ was not detected in cells of myeloid lineage in any of the patients. ${ }^{32}$ Alternatively, transcripts may derive from a small, persistent population of immature but nonclonogenic myeloid precursors that slowly decrease with time. ${ }^{33}$ This could be a consequence of leukemia stem cell exhaustion as demonstrated by Caocci et al., who noted that patients with shorter telomeres in peripheral blood monocytes had a higher chance of successful TFR. ${ }^{34}$ Of note, in another study shorter telomeres at diagnosis were associated with molecular instability and worse overall outcome, possibly indicating that the role of telomeres varies with the stage of disease and might be a dynamic predictive marker. ${ }^{35}$

\section{Success rate of treatment-free remission}

The success rate of TFR has been quite similar across studies from several countries despite the inclusion of patients receiving different TKI and some slight differ- ences in enrollment criteria (Table 1). In addition, time to loss of MMR is very similar across studies with most patients relapsing in the first 6 months. The kinetics of molecular recurrence is similar across all TFR studies with the disease-free status from the STIM 1 study shown as an example in Figure 1. ${ }^{3}$ The molecular recurrence-free survival rate was $38 \%$ at 5 years. It is worth noting that patients who have had undetectable transcripts for many years can relapse so quickly once the suppressive pressure on the residual quiescent stem cells is released.

The DASfree study enrolled patients receiving dasatinib as first- or second-line therapy. The TFR rate at 2 years was slightly higher for patients on first-line dasatinib compared to those given dasatinib as a second-line therapy (51\% vs. 44\%, respectively). ${ }^{7}$ The ENESTfreedom enrolled patients treated with nilotinib. Of the 190 patients who discontinued therapy, 44\% remained in TFR at 192 weeks. The LAST ${ }^{1}$ and EURO-SKI studies ${ }^{6}$ enrolled patients on multiple TKI, with the majority of patients receiving imatinib. In the LAST study, with a median follow-up of 12 months, the molecular relapse-free survival rate was $66 \%$ at 24 months. In the EURO-SKI study with a median follow-up of 24 months, the molecular relapse-free survival was $50 \%$ at 24 months.

\section{Monitoring}

As most molecular recurrences occur in the first 6 months after stopping TKI therapy, more intensive monitoring is recommended earlier after stopping the treatment. Currently, guidelines suggest monitoring with peripheral blood PCR analysis every 4 weeks for 6 to 12 months, then every 6 to 8 weeks for 12 to 18 months, then every 3 months thereafter. Whether this schedule of intensive upfront monitoring is really needed remains unclear and a recent mathematical modeling experiment suggests

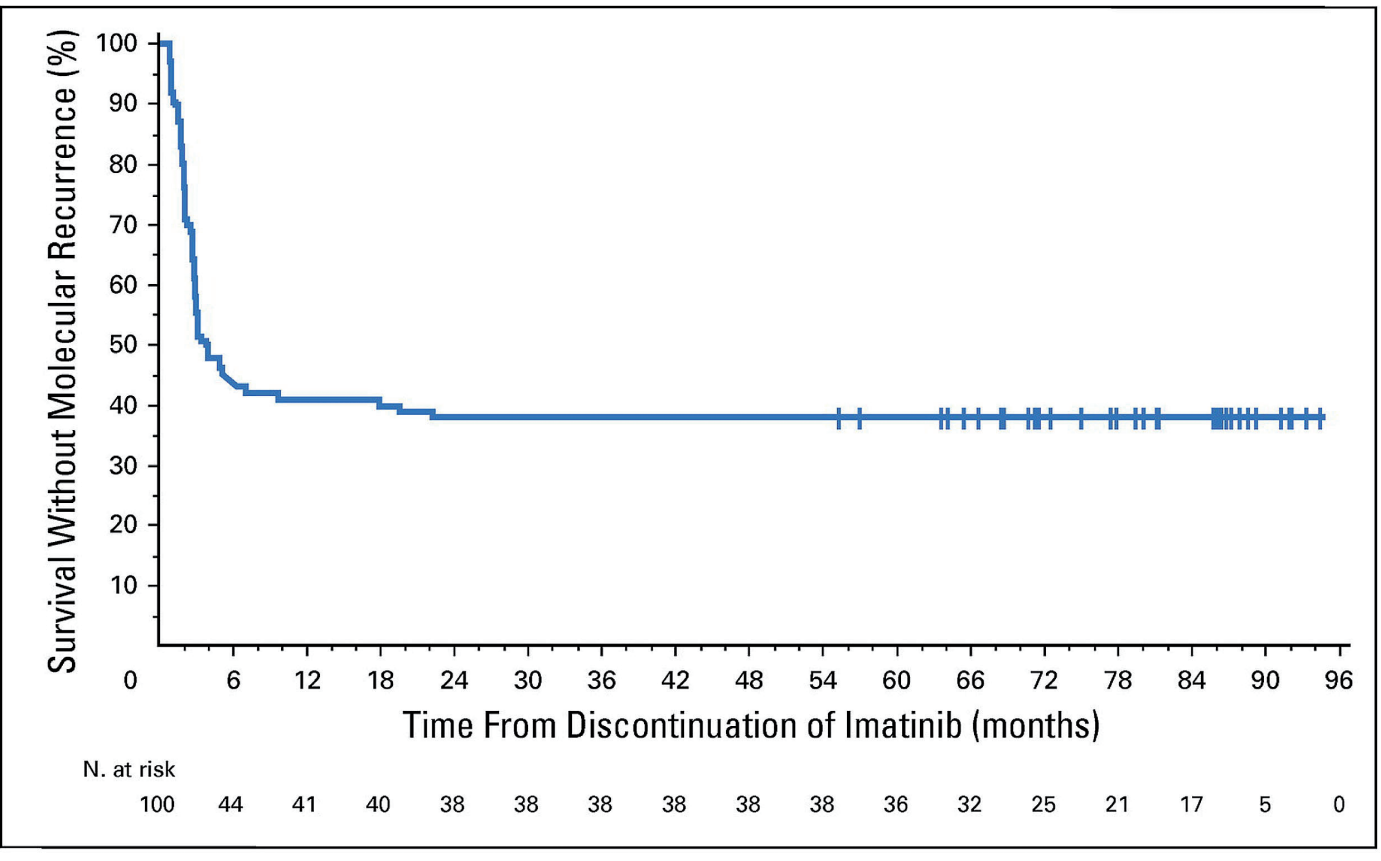

Figure 1. Molecular recurrence-free survival after discontinuation of imatinib in patients $(n=100)$ in the STIM1 study. ${ }^{3}$ 
that initial monitoring every 2 months followed by every 3 months, should transcripts remain undetectable after 6 months, is likely to be a safe approach. ${ }^{36}$ Monthly PCR evaluations increase patients' anxiety and may present an economic hurdle for some patients or countries.

The mechanisms by which the CML clone is either eliminated or perhaps more likely, suppressed are not fully understood. It has been suggested that small amounts of residual disease can be controlled by autologous immune suppression. A number of studies have evaluated T-cell and particularly NK-cell populations in patients in TFR, but none has been convincing and further studies are needed. ${ }^{37,38}$ It is conceivable that residual clonogenic cells remain in a relatively quiescent state which may take years to manifest clinically, perhaps because of an eventual failure of immune control. With few exceptions, we do not know the interval between the original acquisition of the $B C R-A B L 1$ mutation and the development of clinically apparent CML. The median time to the development of "clinical" CML after the atomic bombing of Japan was approximately 10 years with cases continuing to be detected for years after that. ${ }^{39}$ Hence, the importance of long-term, continued PCR monitoring cannot be over emphasized as late relapses have been reported and remain a concern. Rousselot et al. identified nine patients relapsing more than 2 years (median 3.6 years) after stopping, with late relapses seeming to be more common in patients with fluctuating detectable transcript levels during earlier follow-up..$^{40}$

Our policy is to continue to monitor patients about every 4-6 months after 3 years in TFR; it is likely that this approach could be modified with the availability of more long-term follow-up information. It should be noted that PCR standardization and monitoring are not uniformly available around the world and in the USA. It has been reported that approximately $50 \%$ of US physicians do not have adequate PCR monitoring and do not follow guidelines when it comes to TKI discontinuation, so further educational efforts are warranted. ${ }^{41}$

\section{Are there factors that predict successful treatment-free remission?}

The duration of TKI treatment, Sokal risk score, duration of DMR, depth of response, the use of highly sensitive droplet digital PCR (ddPCR), number of NK cells, and type of $B C R-A B L 1$ transcript have all been shown to predict the success rate of TFR to a varying degree in some, but not all studies.

- The duration of TKI treatment has perhaps been the most reproducible predictor of successful therapy discontinuation. TKI therapy for more than 5 years has been associated in multiple studies with higher rates of TFR. For example in the STIM trial, the risk of molecular recurrence was $50 \%$ for those who were on imatinib $>54$ months versus $80 \%$ for those on imatinib $<54$ months. ${ }^{22}$ In the large EURO-SKI trial, the 6-month probability of maintaining a MMR was $63 \%$ for patients on imatinib treatment for $>5.8$ years versus $41 \%$ for those treated for $<5.8$ years. However, when the duration of TKI therapy and duration of DMR were entered into the same model, only one of them was predictive as both these factors were closely correlated. ${ }^{6}$ These observations emphasize the need for many years of treatment before most patients can be considered for discontinuation and should be part of the initial discussion with patients beginning treatment for chronic phase CML. It also indicates that caregivers must be skilled in managing the side effects of TKI therapy so as to maximize compliance.

- The duration of DMR is less consistent in terms of predicting successful TFR. All studies of TKI discontinuation required a minimum of 2 years of documented DMR although the definition of DMR varied across studies (Table 1). The duration of deep remission was not predictive in STIM1, DASfree, ${ }^{7}$ or ENESTfreedom ${ }^{4}$ but was predictive in EURO-SKI. ${ }^{6}$ In the EURO-SKI trial, the 6-month probability of maintaining MMR was $61 \%$ for patients who maintained MR4 for $>3.1$ years versus $44 \%$ for those who maintained MR4 for $<3.1$ years.

- Sokal risk score: a lower score was predictive of better outcomes in ENESTfreedom ${ }^{43}$ and EUROSKI; ${ }^{6}$ however, in everyday practice the Sokal risk score is currently calculated for very few patients, making this a less practical marker. Nonetheless, the rate of TFR is likely to be lower in patients with more "advanced" chronic phase CML.

- Type of transcript: several studies have demonstrated that patients harboring the e14a2 transcript are more likely to achieve a DMR and have successful TFR compared to those with e13a2.44 In one study by D'Adda et al., the rates of a sustained DMR and maintaining TFR were $39.6 \%$ versus $19.4 \%$ and $61 \%$ versus $22 \%$ for patients with e14a2 versus e13a2 transcripts, respectively. ${ }^{45}$

- Depth of response is also emerging as an important factor in predicting successful TFR. In the DESTINY trial, ${ }^{2}$ in which patients with $B C R-A B L 1<0.1 \%$ were enrolled, a dose de-escalation phase was included. After enrollment into the study, patients received $50 \%$ of the FDAapproved dose of their TKI for 1 year. Patients with at least one IS measurement of transcripts between $0.01 \%$ and $0.1 \%$ were considered in the MMR group, while patients who had all measurements $<0.01 \%$ were considered in the MR4 group. At 3 years, the rate of successful TFR was $72 \%$ versus $33 \%$ in the MR4 and MMR groups, respectively. ${ }^{2}$ These results suggest that patients in continuous MMR may consider dose de-escalation or may even consider TKI discontinuation, understanding that the chance of successful TFR is approximately $30 \%$.

The ENESTfreedom study enrolled patients with chronic phase CML who had been on nilotinib for at least 2 years and achieved MR4.5. Patients who maintained MR4 during an additional year of follow-up discontinued the drug. Among the 190 patients who discontinued nilotinib, the rate of successful TFR was $50 \%$ for those who maintained MR 4.5 versus $35 \%$ for those with at least one level $>$ MR $4.5 .{ }^{43}$

ddPCR is $1-2$ logs more sensitive than real-time quantitative $\mathrm{PCR}^{46}$ and some studies have demonstrated that negative or undetectable transcripts by ddPCR are most predictive of successful TFR. ${ }^{47-49}$ In a study by Nicolini et al., of the 174 patients who discontinued therapy and for whom ddPCR results were available, 37 had $B C R-A B L 1$ values $\geq 0.0023 \%$. Loss of MMR occurred in $68 \%$ of patients who had $B C R-A B L 1$ values $\geq 0.0023 \%$, compared to $46 \%$ with levels $<0.0023 \%$. As for patients monitored by real-time quantitative PCR, patients with detectable $B C R-A B L 1$ by ddPCR may still consider a TFR attempt and ddPCR BCR-ABL1 levels may further refine the selection of patients for an attempt at TKI discontinuation. 
Currently ddPCR is not widely available and standardization across laboraties will be required prior to its use in routine clinical care.

All these data suggest that the depth of response matters. The probability of successful TFR is highest for patients with negative ddPCR results, followed by those with MR4.5, then MR4 and finally, MR3 (Table 4). However, this distinction is not absolute, since relapse rates are still considerable at all these levels and patients and physicians may choose to consider a trial of TKI discontinuation at higher transcript levels with the understanding that the risks of molecular recurrence and the need to restart treatment are higher.

- Other investigational studies have associated a higher percentage of innate $\mathrm{T}$ cells ${ }^{37}$ or $\mathrm{NK}$ cells $^{38}$ with a better chance of TFR, suggesting that an immune surveillance mechanism is involved in maintaining molecular response.

\section{Side effects of discontinuation}

As noted in the LAST study, which included a comprehensive evaluation of patient-reported outcomes after TKI discontinuation and at the time of restarting TKI, TKI discontinuation is associated with clinically significant improvements of fatigue, diarrhea, depression and sleep disturbances in many patients. ${ }^{50}$ Conversely, some patients who discontinue TKI and need to restart have significant anxiety ${ }^{51,52}$ with one patient describing it "as having leukemia all over again."

Musculoskeletal pain is a well-recognized side effect of TKI discontinuation. ${ }^{53,54}$ Approximately $30 \%$ of patients have an increase in musculoskeletal pain or periarticular stiffness, which is usually transient. These symptoms can be managed with non-steroidal anti-inflammatory drugs, and occasionally glucocorticosteroids and acetaminophen. Pain is more common in patients with a prior history of musculoskeletal pain and in those who have been on TKI therapy for a long period of time..$^{53} \mathrm{~A}$ small minority of patients need to restart TKI for uncontrolled discomfort. Several mechanisms have been proposed to explain this phenomenon, including activation of other kinase-mediated pathways which had been inhibited by the TKI, such as release of c-kit inhibition, ${ }^{55}$ mast cell activation, ${ }^{56}$ or bone remodeling effects, ${ }^{57}$ but none has been proven yet.

\section{Dose reduction}

For a large majority of patients who cannot achieve TFR, another approach to reduce side effects and costs and to maintain compliance would be to identify the lowest possible effective dose of TKI. ${ }^{58,59}$ Both dasatinib and nilotinib have demonstrated efficacy at doses lower than the FDA-approved dose. In a recently published phase II study from the $\mathrm{MD}$ Anderson Cancer Center, ${ }^{60}$ dasatinib was given at a dose of $50 \mathrm{mg}$ daily, instead of the FDAapproved dose of $100 \mathrm{mg}$, to newly diagnosed patients. Of the 81 patients enrolled, $81 \%$ had achieved a MMR at 12 months according to the follow-up data at the time of the publication. This is higher than in the DASISION study, in which the MMR rate was $46 \%$ at 1 year with dasatinib. ${ }^{24}$ The $50 \mathrm{mg}$ dose was better tolerated with a low cumulative incidence of pleural effusions of $6 \%$.
Table 4. Studies reporting the rate of successful treatment-free remission based on depth of response.

\begin{tabular}{lccc} 
Study & Level & TFR \% & @ \\
Bernardi9 $^{49}$ & ddPCR $<0.468$ & 83 & 2 years \\
& ddPCR $>0.468$ & 52 & 2 years \\
& MR4 & 74 & 2 years \\
& MR $4.5-5$ & 80 & 2 years \\
Nicolini $^{48}$ & ddPCR $<0.0023 \%$ & 68 & 1 year \\
& ddPCR $>0.0023 \%$ & 46 & 1 year \\
\hline ENEST freedom $^{43}$ & MR4 & 35 & 2 years \\
& MR4.5 & 51 & 2 years \\
DESTINY $^{2}$ & $<$ MR4 & 72 & 3 years \\
& MR3 & 33 & 3 years
\end{tabular}

TFR: treatment-free remission; MR: molecular response (with log reduction in $B C R$ ABL1 transcripts); ddPCR: droplet digital polymerase chain reaction analysis.

The NiloRED study was an observational study evaluating dose reductions for patients on nilotinib ${ }^{61}$ Of the 67 evaluable patients, $68.6 \%$ were receiving nilotinib as firstline therapy and $31.4 \%$ as second-line treatment. The median duration of MMR was 25 months at the time of dose reduction. The nilotinib dose was reduced to $450 \mathrm{mg}$ daily, $400 \mathrm{mg}$ daily and $300 \mathrm{mg}$ daily in $87 \%, 10 \%$ and $3 \%$ patients, respectively. Only two patients lost the MMR, although they regained MR4 later on without a dose increase.

The DESTINY trial, detailed earlier, provides a perfect example illustrating the safety of dose reduction. ${ }^{2}$ Of the 174 patients enrolled, 148, 16 and 10 were receiving imatinib, nilotinib and dasatinib, respectively. At study entry, the TKI dose was reduced to half the standard dose for 12 months: imatinib $200 \mathrm{mg}$ daily, dasatinib $50 \mathrm{mg}$ daily, or nilotinib $200 \mathrm{mg}$ twice daily. After 1 year, 19\% of patients in the group with MMR had lost their MMR compared to $2 \%$ of those starting with deeper responses. After 1 year of half-dose therapy, patients who had not "relapsed" discontinued therapy as discussed earlier. It is unclear what would have happened if those patients had stayed on a lower dose and had not attempted treatment discontinuation. The INTERIM trial was another study that demonstrated the safety of imatinib dose reduction for patients in a complete cytogenetic remission (CCyR). ${ }^{62}$ In that study, imatinib was reduced to 1 month on $/ 1$ month off in 67 patients who were $>65$ years of age and had attained CCyR. Of those patients, $17 \%$ and $18 \%$ lost their CCyR and MMR, respectively. All patients who restarted higher dose therapy attained CCyR and MMR again.

In summary, these data demonstrate that, particularly for patients experiencing chronic symptoms, dose reduction can be an option for those on a stable dose of TKI with adequate response, including those who are not eligible for or do not want an attempt at treatment discontinuation, and those in whom previous TKI discontinuation failed. More frequent monitoring is advisable for the first 6 months after dose reduction to detect the unusual individual who rapidly loses response.

In this regard, Fassoni and colleagues, using data from large clinical trials, modeled the kinetics of transcript levels after a $50 \%$ reduction in dose in patients in stable MMR..$^{58}$ Importantly, their model predicts a transient increase in $B C R-A B L 1$ transcripts shortly after dose reduction which decreases to baseline or lower, without a return to the original dose. Thus, the model suggests that clinicians should 
not reflexively increase the dose should the transcript level increase, but rather that they should continue to observe the patient closely. ${ }^{59}$ Indeed, this phenomenon was noted in some patients in the NiloRed trial mentioned above. ${ }^{54}$ Additional prospective, systematic studies of dose reduction would be welcomed in the future.

\section{Future directions}

An attempt at treatment discontinuation should be considered the standard of care for patients with CML who have a sustained DMR. Realistically, only $40-50 \%$ of newly diagnosed patients with CML will achieve this threshold, and only half of those ( $20 \%$ of all patients) will achieve a successful TFR. These estimates are extrapolated from the results of clinical trials and may overstate the actual number of patients treated in general practice who may achieve the required depth and duration of response and be eligible for a trial of TKI discontinuation.
Many unanswered questions remain. How can we better identify patients who will achieve DMR and TFR? Should these patients be considered "cured" of their disease and what are the mechanisms by which the disease is controlled/eliminated? A better understanding of these mechanisms might suggest new approaches to prevent relapse. How can more patients achieve a DMR in order to consider a trial to achieve TFR? The limitations of the currently available TKI have been defined but a new inhibitor ABL001, which inhibits signaling by blocking the myristoyl pocket of the enzyme, ${ }^{63,64}$ has recently been developed and combinations with traditional TKI will be conducted in the hopes of achieving deeper responses as the pathway to higher rates of TFR.

Nonetheless, when the extraordinary results using imatinib first became available, there was little expectation that the responses would be so durable and that it might eventually be possible to avoid lifetime treatment. CML investigators should be proud of these remarkable accomplishments.

\section{References}

1. Atallah E, Schiffer CA, Radich JP, et al. Results from the U.S. Life after Stopping TKIs (LAST) study. Blood. 2017;130(Suppl 1):2903.

2. Clark RE, Polydoros F, Apperley JF, et al. Deescalation of tyrosine kinase inhibitor therapy before complete treatment discontinuation in patients with chronic myeloid leukaemia (DESTINY): a non-randomised, phase 2 trial. Lancet Haematol. 2019;6(7): e375-e83.

3. Etienne G, Guilhot J, Rea D, et al. Long-term follow-up of the French Stop Imatinib (STIM1) study in patients with chronic myeloid leukemia. J Clin Oncol. 2017;35(3): 298-305

4. Giles FJ, Masszi T, Casares MTG, et al. Treatment-free remission (TFR) following frontline (1L) nilotinib (NIL) in patients (pts) with chronic myeloid leukemia in chronic phase (CML-CP): 192-week data from the ENESTfreedom study. J Clin Oncol. 2019;37 (15_suppl):7013.

5. Ross DM, Branford S, Seymour JF, et al. Safety and efficacy of imatinib cessation for CML patients with stable undetectable minimal residual disease: results from the TWISTER study. Blood. 2013, 2013;122(4): 515-522.

6. Saussele S, Richter J, Guilhot J, et al. Discontinuation of tyrosine kinase inhibitor therapy in chronic myeloid leukaemia (EURO-SKI): a prespecified interim analysis of a prospective, multicentre, non-randomised, trial. Lancet Oncol. 2018;19(6): 747-757

7. Shah NP, García-Gutiérrez V, JiménezVelasco A, et al. Dasatinib discontinuation in patients with chronic-phase chronic myeloid leukemia and stable deep molecular response: the DASFREE study. Leuk Lymphoma. 2020;61(3):650-659.

8. Tantiworawit A, Power MM, Barnett MJ, et al. Long-term follow-up of patients with chronic myeloid leukemia in chronic phase developing sudden blast phase on imatinib therapy. Leuk Lymphoma. 2012;53(7):13211326.

9. NCCN guidelines Clinical Practice Guidelines in Oncology. Version 3.2020January 30, 2020. https://www.nccn.org/ professionals/physician_gls [Accessed April 10, 2020].

10. Hochhaus A, Baccarani M, Silver RT, et al. European LeukemiaNet 2020 recommendations for treating chronic myeloid leukemia. Leukemia. 2020;34(4):966-984.

11. Mahon FX, Rea D, Guilhot J, et al. Discontinuation of imatinib in patients with chronic myeloid leukaemia who have maintained complete molecular remission for at least 2 years: the prospective, multicentre Stop Imatinib (STIM) trial. Lancet Oncol. 2010;11(11):1029-1035

12. Atallah E, Schiffer CA, Weinfurt KP, et al. Design and rationale for the Life After Stopping Tyrosine kinase inhibitors (LAST) study, a prospective, single-group longitudinal study in patients with chronic myeloid leukemia. BMC Cancer. 2018;18 (1):359.

13. Mahon F-X, Richter J, Guilhot J, et al. Interim analysis of a pan European stop tyrosine kinase inhibitor trial in chronic myeloid leukemia: the EURO-SKI study. Blood. 2014;124(21):151.

14. Flynn KE, Myers JM, D'Souza A, Schiffer CA, Thompson JE, Atallah E. Exploring patient decision making regarding discontinuation of tyrosine kinase inhibitors for chronic myeloid leukemia. Oncologist. 2019;24(9):1253-1258.

15. Goldberg S, Hamarman S. Patients with chronic myelogenous leukemia may not want to discontinue tyrosine kinase inhibitor therapy. Blood. 2015;126(23): 1584

16. Hughes TP, Boquimpani CM, Takahashi N, et al. Treatment-free remission in patients with chronic myeloid leukemia in chronic phase according to reasons for switching from imatinib to nilotinib: subgroup analysis from ENESTop. Blood. 2016;128(22): 792 .

17. Okada M, Imagawa J, Tanaka H, et al. Final 3 -year results of the dasatinib discontinuation trial in patients with chronic myeloid leukemia who received dasatinib as a second-line treatment. Clin Lymphoma Myeloma Leuk. 2018;18(5):353-360.e1.

18. Rea D, Rousselot P, Guilhot F, et al. Discontinuation of second generation (2G) tyrosine kinase inhibitors (TKI) in chronic phase (CP)-chronic myeloid leukemia (CML) patients with stable undetectable BCR-ABL transcripts. Blood. 2012;120(21): 916.

19. Hughes TP, Boquimpani C, Takahashi N, et al. ENESTop 192-week results: treatmentfree remission (TFR) in patients (pts) with chronic myeloid leukemia in chronic phase (CML-CP) after stopping second-line (2L) nilotinib (NIL). J Clin Oncol. 2019;37 (15 suppl):7005.

20. Kim DDH, Busque L, Forrest DL, et al. Second attempt of TKI discontinuation with dasatinib for treatment-free remission after failing first attempt with imatinib: Treatment-free Remission Accomplished by Dasatinib (TRAD) trial. Blood. 2018;132 (Supplement_1):787.

21. Legros L, Nicolini FE, Etienne G, et al. Second tyrosine kinase inhibitor discontinuation attempt in patients with chronic myeloid leukemia. Cancer. 2017;123(22): 4403-4410.

22. Matsuki E, Sakurai M, Karigane D, et al. Second attempt to discontinue TKI in CML patients who have sustained CMR for over 2 years is rarely successful even with the use of second generation TKIs. Blood. 2016;128(22):1887.

23. Hochhaus A, Saglio G, Hughes TP, et al. Long-term benefits and risks of frontline nilotinib vs imatinib for chronic myeloid leukemia in chronic phase: 5-year update of the randomized ENESTnd trial. Leukemia. 2016;30(5):1044-1054.

24. Cortes JE, Saglio G, Kantarjian HM, et al. Final 5-year study results of DASISION: the dasatinib versus imatinib study in treatment-naïve chronic myeloid leukemia patients trial. J Clin Oncol. 2016;34(20): 2333-2340.

25. Hochhaus A, Masszi T, Giles FJ, et al. Treatment-free remission following frontline nilotinib in patients with chronic myeloid leukemia in chronic phase: results from the ENESTfreedom study. Leukemia. 2017;31(7):1525-1531.

26. Mahon FX, Nicolini FE, Noël M-P, et al. Preliminary report of the STIM2 study: a multicenter stop imatinib trial for chronic phase chronic myeloid leukemia de novo patients on imatinib. Blood. 2013;122 
(21):654.

27. Hughes TP, Leber B, Cervantes F, et al. Sustained deep molecular responses in patients switched to nilotinib due to persistent BCR-ABL1 on imatinib: final ENESTcmr randomized trial results. Leukemia. 2017;31(11):2529-2531.

28. Cole AL, Wood WA Jr, Muluneh B, et al. Comparative safety and health care expenditures among patients with chronic myeloid leukemia initiating first-line imatinib, dasatinib, or nilotinib. JCO Oncol Pract. 2020;16(5):e443-e455.

29. Shih YT, Cortes JE, Kantarjian HM. Treatment value of second-generation BCRABL1 tyrosine kinase inhibitors compared with imatinib to achieve treatment-free remission in patients with chronic myeloid leukaemia: a modelling study. Lancet Haematol. 2019;6(8):e398-e408.

30. Rousselot P, Charbonnier A, Cony-Makhoul $\mathrm{P}$, et al. Loss of major molecular response as a trigger for restarting tyrosine kinase inhibitor therapy in patients with chronicphase chronic myelogenous leukemia who have stopped imatinib after durable undetectable disease. J Clin Oncol. 2014;32(5): 424-430.

31. Ross DM, Branford S, Seymour JF, et al. Patients with chronic myeloid leukemia who maintain a complete molecular response after stopping imatinib treatment have evidence of persistent leukemia by DNA PCR. Leukemia. 2010;24(10):17191724.

32. Pagani IS, Dang P, Saunders VA, et al. Lineage of measurable residual disease in patients with chronic myeloid leukemia in treatment-free remission. Leukemia. 2020; 34(4):1052-1061

33. Ross DM, Pagani IS, Shanmuganathan N, et al. Long-term treatment-free remission of chronic myeloid leukemia with falling levels of residual leukemic cells. Leukemia. 2018:32(12):2572-2579

34. Caocci G, Greco M, Delogu G, et al. Telomere length shortening is associated with treatment-free remission in chronic myeloid leukemia patients. J Hematol Oncol. 2016;9(1):63.

35. Wenn K, Tomala L, Wilop S, et al. Telomere length at diagnosis of chronic phase chronic myeloid leukemia (CML-CP) identifies a subgroup with favourable prognostic parameters and molecular response according to the ELN criteria after 12 months of treatment with nilotinib. Leukemia. 2015;29(12):2402-2404

36. Shanmuganathan N, Braley JA, Yong ASM, et al. Modeling the safe minimum frequency of molecular monitoring for CML patients attempting treatment-free remission. Blood. 2019:134(1):85-89.

37. Cayssials E, Jacomet F, Piccirilli N, et al. Sustained treatment-free remission in chronic myeloid leukaemia is associated with an increased frequency of innate CD8(+) Tcells. Br J Haematol. 2019;186(1):54-59.

38. Ilander M, Olsson-Stromberg U, Schlums H, et al. Increased proportion of mature NK cells is associated with successful imatinib discontinuation in chronic myeloid leukemia. Leukemia. 2017;31(5):1108-1116.

39. Hsu W-L, Preston DL, Soda M, et al. The Incidence of leukemia, lymphoma and multiple myeloma among atomic bomb survivors: 1950-2001. Radiat Res. 2013;179(3): 361-382.

40. Rousselot P, Loiseau C, Delord M, Cayuela IM, Spentchian M. Late molecular recurrences in patients with chronic myeloid leukemia experiencing treatment-free remission. Blood Adv. 2020:4(13):3034-3040.

41. Atallah EL, Sadek I, Cao X, et al. Tyrosine kinase inhibitor therapy discontinuation in clinical practice in chronic myeloid leukemia - a US physician survey conducted after guideline updates. Blood. 2019;134 (Suppl_1):2208.

42. Etienne G, Nicolini NE, Dulucq S, et al. Achieving a complete molecular remission under imatinib therapy is associated with a better outcome in chronic phase chronic myeloid leukaemia patients on imatinib frontline therapy. Blood. 2012;120(21):3754 .

43. Ross DM, Masszi T, Gómez Casares MT, et al. Durable treatment-free remission in patients with chronic myeloid leukemia in chronic phase following frontline nilotinib: 96-week update of the ENESTfreedom study. J Cancer Res Clin Oncol. 2018;144(5): 945-954

44. Baccarani M, Rosti G, Soverini S. Chronic myeloid leukemia: the concepts of resistance and persistence and the relationship with the BCR-ABL1 transcript type. Leukemia. 2019:33(10):2358-2364.

45. D'Adda M, Farina M, Schieppati F, et al. The e13a2 BCR-ABL transcript negatively affects sustained deep molecular response and the achievement of treatment-free remission in patients with chronic myeloid leukemia who receive tyrosine kinase inhibitors. Cancer. 2019;125(10):1674-1682.

46. Jennings LJ, George D, Czech J, Yu M, Joseph L. Detection and quantification of BCR-ABL1 fusion transcripts by droplet digital PCR. J Mol Diagn. 2014;16(2):174-179.

47. Mori S, Vagge E, le Coutre P, et al. Age and dPCR can predict relapse in CML patients who discontinued imatinib: the ISAV study. Am J Hematol. 2015:90(10):910-914.

48. Nicolini FE, Dulucq S, Boureau L, et al. Evaluation of residual disease and TKI duration are critical predictive factors for molecular recurrence after stopping imatinib firstline in chronic phase CML patients. Clin Cancer Res. 2019;25(22):6606-6613.

49. Bernardi S, Malagola M, Zanaglio C, et al. Digital PCR improves the quantitation of DMR and the selection of CML candidates to TKIs discontinuation. Cancer Med. 2019;8(5):2041-2055.

50. Flynn KE, Weinfurt KP, Lin L, et al. Patientreported outcome results from the U.S. Life after Stopping TKIs (LAST) study in patients with chronic myeloid leukemia. Blood. 2019;134(Suppl_1):705.

51. Sogawa R, Kimura S, Yakabe R, et al. Anxiety and depression associated with tyrosine kinase inhibitor discontinuation in patients with chronic myeloid leukemia. Int
J Clin Oncol. 2018;23(5):974-979.

52. Sharf G, Marin C, Bradley JA, et al Treatment-free remission in chronic myeloid leukemia: the patient perspective and areas of unmet needs. Leukemia. 2020;34(8):2102-2112.

53. Berger MG, Pereira B, Rousselot P, et al Longer treatment duration and history of osteoarticular symptoms predispose to tyrosine kinase inhibitor withdrawal syndrome. Br J Haematol. 2019;187(3):337-346.

54. Diab M, Schiffer CA. The spectrum of musculoskeletal symptoms in patients with chronic myeloid leukemia after stopping tyrosine kinase inhibitors. Leuk Res. 2019;79:1-2

55. Ceko M, Milenkovic N, le Coutre P, Westermann J, Lewin GR. Inhibition of c-Kit signaling is associated with reduced heat and cold pain sensitivity in humans. Pain 2014;155(7):1222-1228

56. Ramanujam D, McNicholl F, Furby D, et al. Dramatic resolution of respiratory symptoms with imatinib mesylate in patients with chronic myeloid leukemia presenting with lower airway symptoms resembling asthma. Leuk Lymphoma. 2009;50(10): 1721-1722.

57. Breccia M, Alimena G. The metabolic consequences of imatinib mesylate: changes on glucose, lypidic and bone metabolism. Leuk Res. 2009;33(7):871-875

58. Fassoni AC, Baldow C, Roeder I, Glauche I. Reduced tyrosine kinase inhibitor dose is predicted to be as effective as standard dose in chronic myeloid leukemia: a simulation study based on phase III trial data. Haematologica. 2018;103(11):1825-1834.

59. Schiffer IT, Schiffer CA. To what extent can mathematical modeling inform the design of clinical trials? The example of safe dose reduction of tyrosine kinase inhibitors in responding patients with chronic myeloid leukemia. Haematologica. 2018;103(11): 1756-1757.

60. Naqvi K, Jabbour E, Skinner J, et al. Longterm follow-up of lower dose dasatinib (50 $\mathrm{mg}$ daily) as frontline therapy in newly diagnosed chronic-phase chronic myeloid leukemia. Cancer. 2020;126(1):67-75.

61. Rea D, Cayuela J-M, Dulucq S, Etienne G. Molecular responses after switching from a standard-dose twice-daily nilotinib regimen to a reduced-dose once-daily schedule in patients with chronic myeloid leukemia: a real life observational study (NILO-RED) Blood. 2017:130(Suppl_1):318.

62. Russo D, Martinelli G, Malagola M, et al Effects and outcome of a policy of intermittent imatinib treatment in elderly patients with chronic myeloid leukemia. Blood 2013;121(26):5138-5144

63. Hughes TP, Mauro MJ, Cortes JE, et al. Asciminib in chronic myeloid leukemia after ABL kinase inhibitor failure. N Engl J Med. 2019:381(24):2315-2326

64. Schoepfer J, Jahnke W, Berellini G, et al Discovery of asciminib (ABL001), an allosteric inhibitor of the tyrosine kinase activity of BCR-ABL1. J Med Chem. 2018;61(18):8120-8135 